\title{
Natural variation in Fc glycosylation of HIV-specific antibodies impacts antiviral activity
}

\author{
Margaret E. Ackerman, ${ }^{1}$ Max Crispin, ${ }^{2}$ Xiaojie Yu,2 Kavitha Baruah, ${ }^{2}$ Austin W. Boesch, ${ }^{1}$ \\ David J. Harvey, ${ }^{2}$ Anne-Sophie Dugast, ${ }^{3}$ Erin L. Heizen, ${ }^{4}$ Altan Ercan, ${ }^{5}$ Ickwon Choi, ${ }^{6}$ \\ Hendrik Streeck, ${ }^{3}$ Peter A. Nigrovic, ${ }^{7}$ Chris Bailey-Kellogg, ${ }^{6}$ Chris Scanlan, ${ }^{2}$ and Galit Alter ${ }^{3}$ \\ ${ }^{1}$ Thayer School of Engineering, Dartmouth College, Hanover, New Hampshire, USA. ${ }^{2}$ Department of Biochemistry, University of Oxford, \\ Oxford, United Kingdom. ${ }^{3}$ Ragon Institute of MGH, MIT, and Harvard University, Charlestown, Massachusetts, USA. \\ ${ }^{4}$ Center for Human Variation and Department of Medicine, Duke University School of Medicine, Durham, North Carolina, USA. \\ ${ }^{5}$ Division of Rheumatology, Immunology, and Allergy, Brigham and Women's Hospital, Boston, Massachusetts, USA. ${ }^{6}$ Department of Computer Science, \\ Dartmouth College, Hanover, New Hampshire, USA. ${ }^{7}$ Division of Rheumatology, Immunology, and Allergy, \\ Brigham and Women's Hospital, Boston, Massachusetts, USA.
}

\begin{abstract}
While the induction of a neutralizing antibody response against HIV remains a daunting goal, data from both natural infection and vaccine-induced immune responses suggest that it may be possible to induce antibodies with enhanced Fc effector activity and improved antiviral control via vaccination. However, the specific features of naturally induced HIV-specific antibodies that allow for the potent recruitment of antiviral activity and the means by which these functions are regulated are poorly defined. Because antibody effector functions are critically dependent on antibody Fc domain glycosylation, we aimed to define the natural glycoforms associated with robust Fc-mediated antiviral activity. We demonstrate that spontaneous control of HIV and improved antiviral activity are associated with a dramatic shift in the global antibody-glycosylation profile toward agalactosylated glycoforms. HIV-specific antibodies exhibited an even greater frequency of agalactosylated, afucosylated, and asialylated glycans. These glycoforms were associated with enhanced Fc-mediated reduction of viral replication and enhanced $\mathrm{Fc}$ receptor binding and were consistent with transcriptional profiling of glycosyltransferases in peripheral B cells. These data suggest that B cell programs tune antibody glycosylation actively in an antigen-specific manner, potentially contributing to antiviral control during HIV infection.
\end{abstract}

\section{Introduction}

Despite the recent identification of novel monoclonal antibodies with exceptionally broad neutralization potencies, such neutralizing responses have been remarkably difficult to induce via vaccination. However, results from the RV144 vaccine trial, in which protection from infection was observed in $31 \%$ of vaccinees in the absence of neutralizing antibody responses and cytotoxic $T$ cell responses, have reenergized interest in nonneutralizing antibody responses against HIV infection $(1,2)$. Beyond neutralization, antibodies are able to mediate a variety of additional effector functions through their capacity to recruit the innate immune system via $\mathrm{Fc}$ receptors (FcRs). Moreover, these antibodies are readily induced early in HIV infection, are enriched in long-term nonprogressors, and have been shown to provide protection in some models (3-7). However, the specific antibody characteristics that are associated with enhanced innate immune activity have yet to be defined. Based on strong genetic and clinical data from antibody therapeutics, as well as passive transfer and challenge studies in HIV, recruitment of innate immunity is a key factor in antibody activity in vivo, and, therefore, understanding these characteristics is likely to be important for vaccine development efforts.

Conflict of interest: The authors have declared that no conflict of interest exists.

Note regarding evaluation of this manuscript: Manuscripts authored by scientists associated with Duke University, The University of North Carolina at Chapel Hill, Duke-NUS, and the Sanford-Burnham Medical Research Institute are handled not by members of the editorial board but rather by the science editors, who consult with selected external editors and reviewers.

Citation for this article: J Clin Invest. 2013;123(5):2183-2192. doi:10.1172/JCI65708.
The ability of antibodies to recruit innate immune effector cells is tunable, both in terms of the spectrum of innate immune cells recruited and the responses induced, ranging from proinflammatory to antiinflammatory depending on the specific FcRs engaged (8). Several antibody features determine innate immune recruiting capacity, including antibody titer, affinity, epitope specificity, and polyclonality, each playing a significant role in effector function by impacting the geometry and valency of the immune complexes formed. Because many of the innate immune receptors for antibodies are of low affinity, avid interactions are required to create multivalent immune complexes to cluster receptors and drive cellular activation (9). Furthermore, because these innate receptors are expressed on cellular surfaces, spatial arrangement of both antibody and receptor can have an impact on recognition and induction of effector functions $(10,11)$.

Beyond these variable domain features that modulate the potency of the humoral immune response, antibodies provide instructions to the innate immune system on how to clear complexed antigens via their Fc domain, providing an additional level of regulated control over antibody activity. Despite its nomenclature, the constant domain $(\mathrm{Fc})$ of an antibody possesses a large number of possible states with regard to antibody potency. The 4 subclasses of $\operatorname{IgG}$ (IgG1, IgG2, IgG3, IgG4) vary somewhat in amino acid sequence but dramatically in their ability to bind innate immune receptors (12). Furthermore, within a given subclass, the inflammatory properties are more finely regulated by the specific glycan incorporated on Asn297 of the 
heavy chain, which may be 1 of > 30 sugar structures that greatly influences the affinity between IgG and FcRs or complement proteins (13). Glycosylation of the Fc domain critically modulates the ability of an antibody to interact with FcRs - permitting bidirectional control and tuning of an antibody's inflammatory or antiinflammatory activity and selective engagement of particular innate effector cell activities.

Global antibody glycosylation is altered in numerous disease states, and these alterations can be highly functionally relevant, as changes in fucose and sialic acid content can lead to a thousandfold improvement in the antibody-dependent cellular cytotoxicity (ADCC) activity or, conversely, grant antibodies antiinflammatory properties $(14,15)$. While a complete structure/function map of antibody glycans is lacking, the presence or absence of 3 specific sugar residues on this $\mathrm{N}$-linked biantennary glycan dramatically modulates antibody interactions with FcR. Fucosylation of the mannose core impacts recognition by the activating FCGR3A (14); sialylation of terminal galactose groups is associated with antiinflammatory activity and reduced $\mathrm{F} \gamma \mathrm{R}$ binding (15); and decreased galactosylation has been implicated in altered interactions with complement proteins (16). Previous work has shown that chronic progressive HIV infection is associated with an enrichment of antibodies with agalactosylated (G0) glycans (17), also associated with autoimmune relapse or flares (18).

The N-linked glycans found at Asn297 can be divided into 3 major classes: (a) G0 structures, which are agalactosylated and associated with inflammation; (b) G1 structures, which are singly galactosylated species abundant in healthy individuals; and (c) G2 structures, which possess 2 galactose groups and are associated with antiinflammatory activity, as they have an increased potential to be sialylated.

While neutralizing antibody responses emerge in only a fraction of HIV-infected individuals with progressive disease, innate immune recruiting antibodies, such as those able to elicit ADCC or, more broadly, antibody-dependent cellular viral inhibition (ADCVI) emerge in the majority of individuals able to spontaneously control HIV infection $(3,4)$. These data point to a potential role of these humoral immune responses in the control of infection. However, little is known about the biophysical properties of naturally produced antibodies that are able to potently recruit the antiviral activity of the innate immune system, and less is known about the degree of antibody glyco-variation in the context of infection, in which naturally induced glycovariants contribute most profoundly to antiviral control, or whether glyco-variation is actively harnessed by the immune system naturally to gain more effective control of infection. Here we report that while HIV infection is marked by a bulk antibody shift toward glycans associated with inflammation, spontaneous control of HIV is associated with the production of antigen-specific antibodies with unique glycoforms superbly tuned to recruit the antiviral activity of the innate immune system. These data suggest that unique antigen-specific B cell programs can result in skewed antibody glycosylation, which may contribute to potent antiviral control during progressive HIV infection.

\section{Results}

Antiviral effector activity of antibodies from HIV-infected subjects. To begin to define the specific humoral properties associated with the antiviral activity of innate immune recruiting antibodies, antibodies from patients with differential disease progression were isolated from plasma and used in an ADCVI assay $(19,20)$, which measures both cytolytic and noncytolytic mechanisms of antiviral activity. In this long-term assay, neutralizing activity against this tier 2 virus was rare in all subject groups (Figure 1A). However, antibodies from elite controllers and chronic patients on therapy elicited the antiviral activity of NK cells to inhibit viral replication more potently than antibodies from untreated chronic progressors (Figure 1A), implying that low-level viral replication may be associated with the induction of more potent innate immune recruiting antibody responses. Additionally, antibodies from viral controllers induced more robust NK cell responses, with a more multifunctional profile and greater extent of degranulation (Figure $1 \mathrm{~B})$, suggesting that even among subjects with potent inhibition of viral outgrowth, qualitative differences in the innate immune recruiting capacity of humoral immune responses exist.

In order to account for the mechanism of this differential activity, each of the 3 antibody features known to modulate innate immune recruiting potential was investigated. No differences were observed in antibody titer or IgG subclass distribution in either bulk or HIV-specific antibodies among the different patient populations (data not shown), consistent with previous reports (21), suggesting that these features could not account for the differences observed in ADCVI activity. In contrast, enzymatic removal of the glycan from the antibody heavy chain resulted in profound loss of ADCVI activity (Figure 1C).

Global antibody glycosylation is altered by HIV infection. Thus, to determine whether specific changes in the distribution of antibody glycan structures could be associated with differences in the magnitude of ADCVI activity observed among patient groups, the distribution of glycoforms among the different patient populations was compared. The $\mathrm{N}$-glycans were identified by negative-ion electrospray collision-induced fragmentation (ESI-CID) mass spectrometry, and their relative proportions were measured by MALDI-TOF mass spectrometry. The identified glycans were complex biantennary species, with and without fucose and/or a "bisecting" GlcNAc (attached to the 4 position of the branching mannose) and with 0,1 , or 2 galactose residues. The structures of the glycans are outlined in ref. 22 and Supplemental Figure 1 (supplemental material available online with this article; doi:10.1172/JCI65708DS1).

Interestingly, we observed a marked shift in the proportion of agalactosylated glycan species on bulk antibodies in infected subjects compared with that in healthy controls (Figure 1D). This expansion of G0 glycans occurred at the expense of G1 and G2 class glycans, which, as expected, were prevalent forms in the healthy HIV-negative controls. Surprisingly, perturbation of global plasma IgG glycosylation was apparent even during acute infection, indicating that despite the long plasma half-life of IgG, infection rapidly impacts the composition, and therefore activity, of the antibody compartment. Furthermore, although elite controllers durably maintain HIV replication to undetectable levels and exhibit low-level systemic immune activation $(23,24)$, the shift toward agalactosylated glycans was even more pronounced in these individuals compared with that in both treated and untreated chronically infected subjects (Figure 1D), suggesting that unlike the $\mathrm{T}$ cell compartment, the $\mathrm{B}$ cell compartment in elite controllers is inflamed (25), resulting in the production of antibodies with altered glycan composition. However, despite this change, the increase in G0 could not explain the difference in ADCVI activity among infected subject groups. 
A

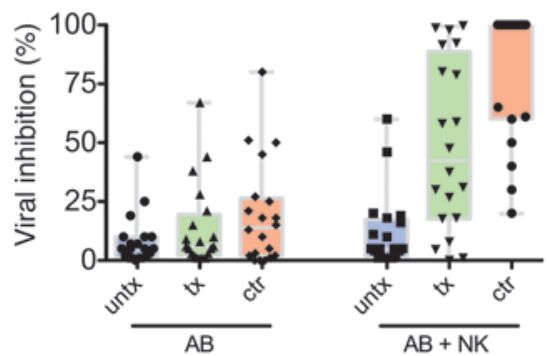

D

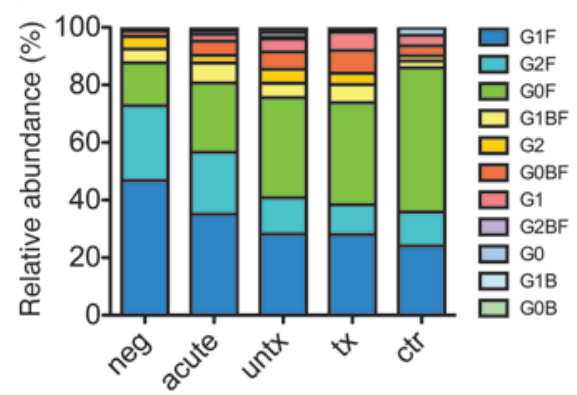

B

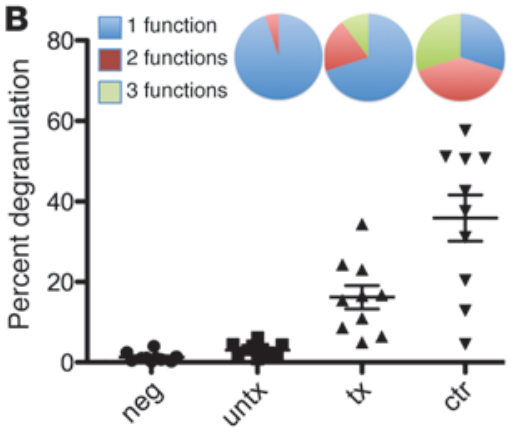

C

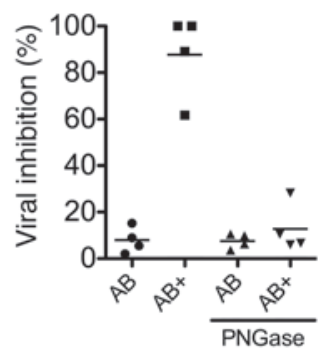

Figure 1

Effector function and global antibody glycosylation profiles. (A) Antibodies from HIV-infected subjects were examined for their ability to inhibit viral replication via neutralization $(A B)$ or ability to recruit NK cells as effectors (NK $+A B)$ in an $A D C V I$ assay. Colored bars correspond to unique glycan structures. (B) NK cell degranulation (dot plot) and cytokine secretion were determined in the ADCVI assay. Pie charts depict the polyfunctional profile of antibody-mediated NK cell recruitment, including degranulating NK cells (CD107a, 1 function), IFN- $\gamma$ secretion plus degranulation (2 functions), or IFN- $\gamma$ plus TNF- $\alpha$ plus degranulation (3 functions). (C) Glycan dependence of ADCVI activity. Removal of antibody glycan with the endoglycosidase PNGaseF did not affect neutralization (AB), but ablated viral inhibition resulting from recruitment of NK cells as effectors $(A B+)$. (A-C) Individual symbols represent each subject tested, and horizontal lines represent the median for a given group of clinically related subjects. (D) Global plasma IgG glycosylation profiles for all glycan structures (first panel), galactose content (second panel), and fucose content (third panel). neg, healthy controls; acute, acutely HIV-infected subjects; untx, untreated chronic progressors; tx, treated chronic progressors; ctr, controllers; F, fucosylated; nonF, nonfucosylated.

Because core $\alpha 1-6$-linked fucose has been implicated in potently modulating ADCC activity via altered affinity for FCGR3A, an activating Fc $\gamma$ R expressed on NK and other innate cells $(14,26)$, we next sought to determine whether lack of fucose was associated with enhanced antibody effector activity and compared the overall levels of fucosylated and nonfucosylated antibodies among patient populations. While no difference was observed in the overall frequency of nonfucosylated glycoforms (Figure 1D), deconvolution of the fucosylated and nonfucosylated forms of each of the G0, G1, and G2 glycan forms revealed some differences in the frequency of nonfucosylated and branched glycan species among subject groups (Figure 2).

HIV-specific antibodies exhibit potentiated glycan skewing. To gain refined insight into the glycan changes specifically associated with the improved innate immune recruiting activity of antibodies from controllers and treated subjects observed in ADCVI assays, antigen-specific antibodies were affinity purified, and the glyco-variation on envelope-specific antibodies, likely involved in mediating this antiviral function, was determined. Significantly, the HIV-specific antibody compartment in all 3 patient groups showed evidence of both inflammation and increased inflammatory activity, exhibiting increases in nonfucosylated, G0, and G1 glycans, and decreases in the less activating, fucosylated G2F and G1F glycans (Figure 3A). Several subjects exhibited remarkable lev- els of nonfucosylated HIV-specific antibodies, with greater than $50 \%$ of envelope-specific antibodies lacking fucose. Additionally, there was a trend toward reduced sialylation of the HIV-specific antibodies ( $P=0.09$, unpaired $t$ test; data not shown). This shift toward decreased fucose, galactose, and sialic acid on HIV-specific antibodies is consistent with a mechanism controlling glycosylation on an antigen-specific basis, rather than simply via global inflammatory signals.

Despite their significant activity differences, only minor differences in the total galactose or fucose content of HIV-specific antibodies were observed among subject groups, although agalactosylated glycans were somewhat enriched in the HIV-specific antibodies of elite controllers (Figure 3B).

However, when comparisons were made as to differences observed in bulk and HIV-specific antibodies within each subject group, controllers clearly exhibited more robust glyco-variation than chronic progressors (Supplemental Figure 2). Relative to bulk plasma IgG, the inflammatory nonfucosylated, G0, and G1 glycoforms were significantly enriched only in elite controllers, and antiinflammatory sialylated and G1F glycoforms were reduced (Figure 4A), consistent with the increased ADCVI activity observed in this population and indicative of more robust antigen-specific modulation of glycan in these subjects. Additionally, when HIV-specific antibody glycans were compared among sub- 


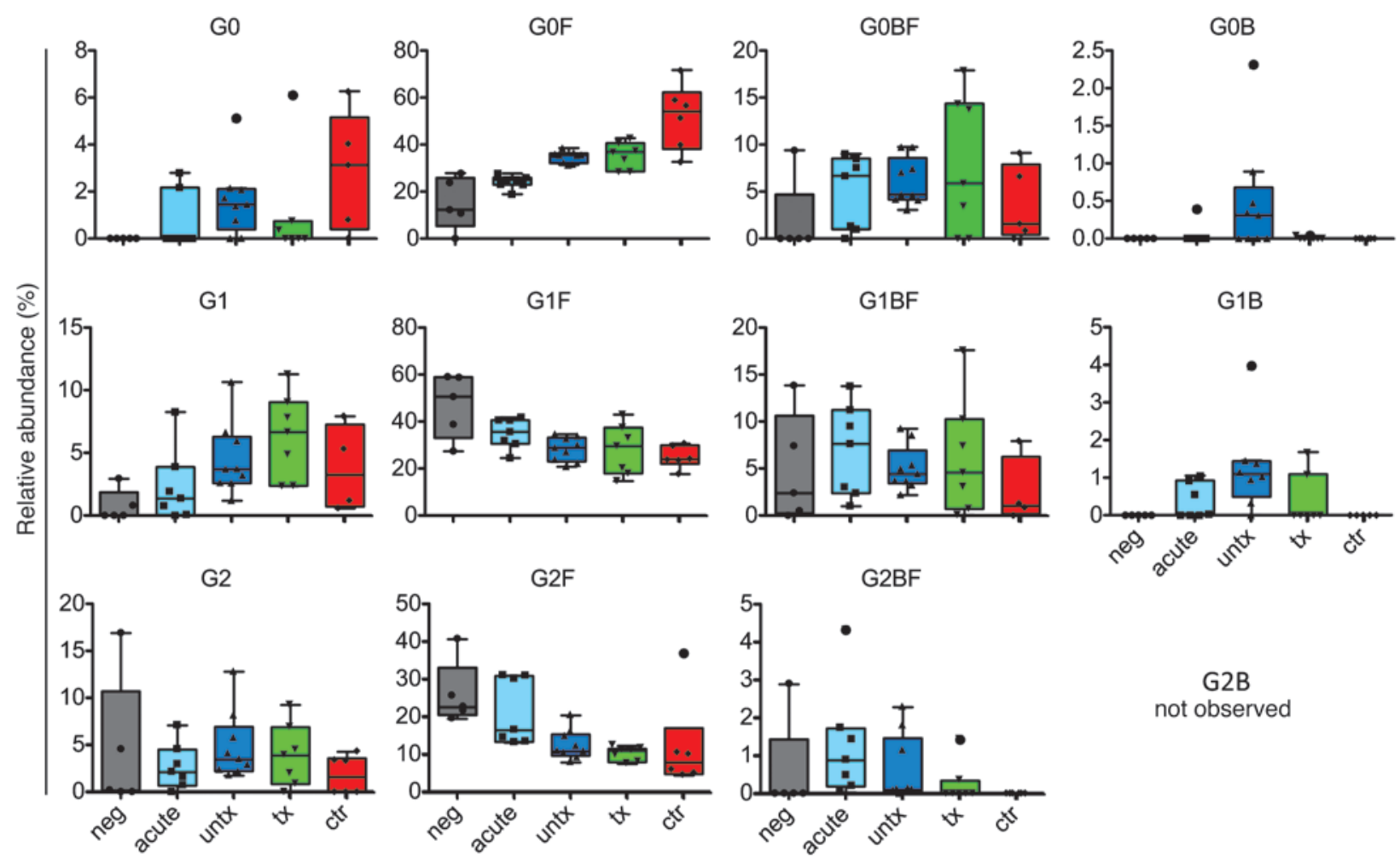

Figure 2

Complete glycan prevalence data for bulk plasma IgG. Relative prevalences (\%) of all observed glycoforms in HIV-negative, acute-infected, treated, and untreated subjects and HIV controllers. Colored underlays represent Tukey's bars and whiskers. In box-and-whisker plots, horizontal bars indicate the medians, boxes indicate 25th to 75th percentiles, and whiskers indicate 10th and 90th percentiles.

ject groups, elite controllers possessed significantly lower levels of G1F, G2F, and bigalactosylated glycans and higher levels of agalactosylated glycoforms on HIV-specific antibodies compared with untreated chronic progressors (Figure 4B). Because galactosylated glycoforms have the potential to be sialylated, these alterations in galactose content may covary with sialylation. Indeed, the trend toward reduced sialylation observed in controllers is consistent with such covariance, as can be readily observed in bulk glycans (Supplemental Figure 3).

Last, relative to uninfected controls, bisected glycans, previously associated with reduced incorporation of fucose and therefore exhibiting higher ADCC (27), were enriched in all infected subject groups, and this enrichment was most profound in treated subjects (Figure 2 and Figure 4B), potentially conferring an alternate route by which treated patients may regain some capacity to recruit NK cell-mediated antiviral control (Figure 1A). These data suggest that progressive HIV disease, marked by high-level viral replication, either persistently or prior to the initiation of antiretroviral therapy, results in a generalized production of more bisected glycans on both HIV-specific and total immunoglobulins and may offer an alternative strategy by which treated chronic progressors explore glycan structures to enhance ADCVI/ADCC activity.

Antibody galactosylation is associated with antiviral activity. Enrichment of agalactosylated glycans was associated with increased ADCVI, whereas bigalactosylated glycans were associated with reduced effector function (Figure 5, A and B, respectively). As a negative control, neutralization activity did not correlate with any glycan structures (data not shown). Additionally, ADCVI activity correlated positively with binding to FCGR3A (Figure 5C). Despite the well-characterized affinity improvement of nonfucosylated glycans to FCGR3A, the correlation between ADCVI activity and the abundance of nonfucosylated glycoforms did not reach statistical significance, indicating that in the context of naturally raised antibodies, galactose levels may play a more significant role in ADCVI activity than the presence or absence of fucose. Because reduced galactosylation has not been associated with potentiated ADCC activity in monoclonal antibodies, the association observed here may reflect concomitant variations in the overall glycan structure, such as altered sialylation, as is implied by the reduced sialylation of HIV-specific antibodies and a relationship between galactose and sialic acid content (Supplemental Figure 3). However, others have shown that antibody affinity purification of IgG on FCGR3A resin favored G0 glycan enrichment by $10 \%$, whereas both G1 and G2 glycoforms were decreased by $10 \%$ and $20 \%$, respectively (28), consistent with a FCGR3A preference for G0 antibody glycans. Moreover, to confirm the biophysical interaction of agalactosylated glycans with FCGR3A, antibody samples from 4 subjects were enzymatically treated with a galactosidase, resulting in increased G0 glycan and decreased G2 content (Figure $5, \mathrm{D}$ and $\mathrm{E}$ ). When the ability of these antibodies to bind to FCGR3A conjugated to a Luminex bead was assessed, the enzyme-treated pool exhibited significantly higher binding (Figure 5F), whereas no impact on FcgR1 binding was observed (data not shown), strongly supporting a preferential physical interaction between FCGR3A and agalactosylated antibodies. 
A

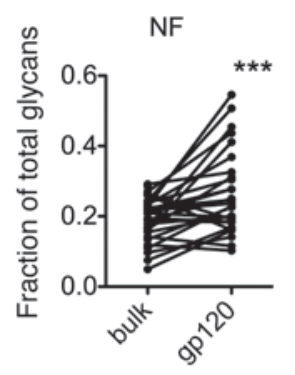

G0

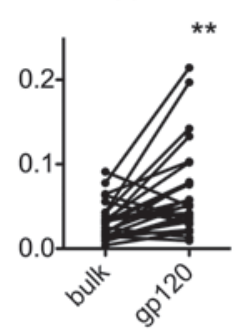

B

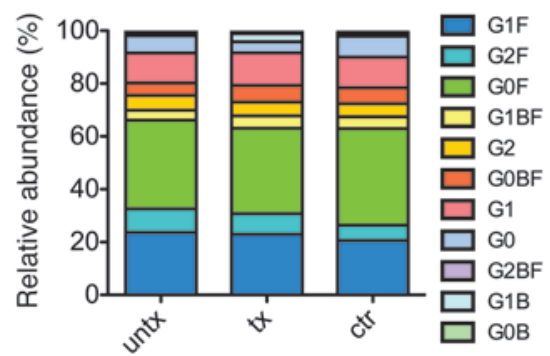

G1

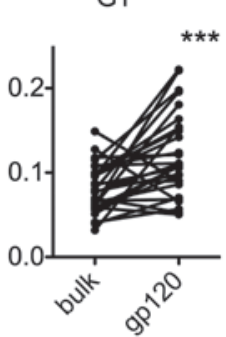

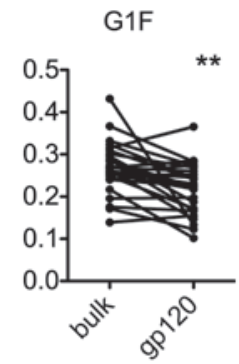

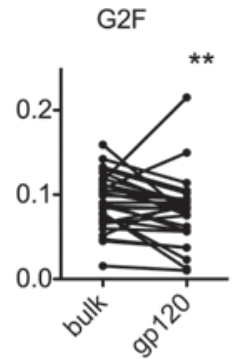

\section{Figure 3}

HIV-specific antibodies exhibit dramatically inflammatory glycosylation profiles. (A) Relative prevalence of glycans on bulk plasma and antigen-specific antibodies. Statistical analysis was performed using Wilcox matched-pairs signed-rank test. NF, nonfucosylated. ${ }^{* \star} P<0.005$; ${ }_{* \star *} P<0.0005$. (B) Glycosylation profiles of HIV-specific antibodies for all glycan structures (first panel), galactose content (second panel), and fucose content (third panel).

B cell glycosyltransferase expression is consistent with antibody glycan alterations. To probe the mechanism underlying differential glycosylation among these subject groups, transcriptional profiling of glycosyltransferases (GTs) and glycosidases associated with the antibody glycan was performed. Expression of 16 GTs (Supplemental Table 2) that may be involved in the biosynthesis of $\mathrm{N}$-linked glycans was determined. When a classifier was trained with 5-fold cross-validation using this data set, it was able to use GT expression data to correctly predict whether a subject was infected or uninfected, averaging better than $85 \%$ accuracy. When applied to further distinguish among infected classes, a classifier differentiated among controllers, untreated subjects, and HIV-negative individuals with better than $70 \%$ accuracy on average but poorly resolved treated subjects and assigned the majority of these subjects to the controller class (data not shown). Indeed, the similarity in GT profiles between treated subjects and controllers is consistent with the glycosylation data reported here. Untreated subjects expressed higher levels of GT transcripts associated with
A

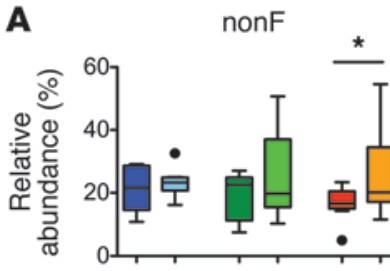

B

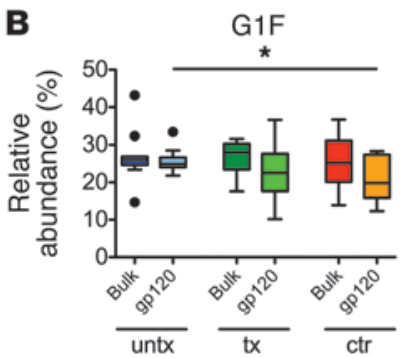

Sialylated

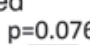

G0
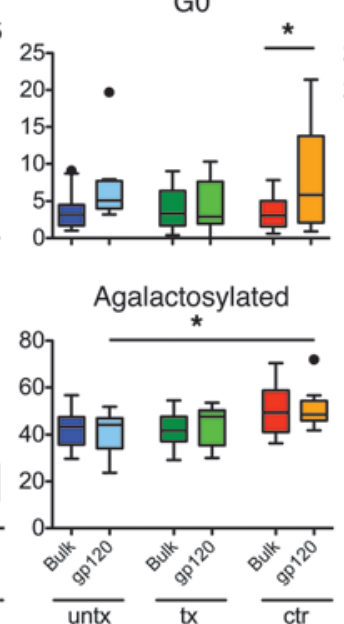

G1
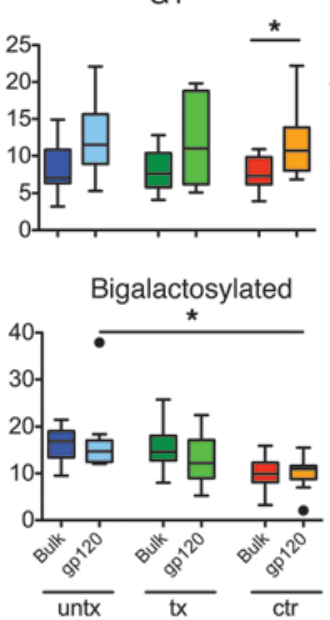

G1F
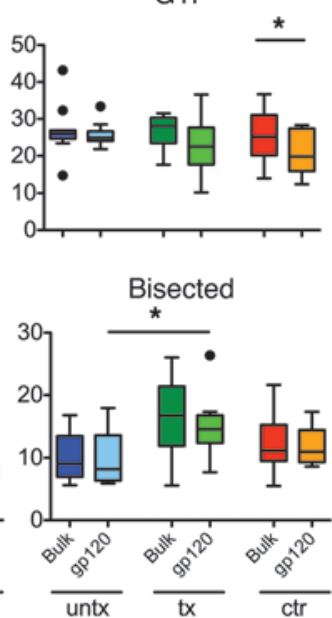

Figure 4

Comparative glycan profiles of bulk plasma and HIV-specific antibodies in each HIV-positive subject class. (A) Significant differences observed between bulk and HIV-specific antibodies within each subject group. (B) Significant differences observed in HIV-specific antibodies among subject groups. In box-and-whisker plots, horizontal bars indicate the medians, boxes indicate 25th to 75th percentiles, and whiskers indicate 10th and 90th percentiles. Dots outside of box plots represent outliers. ${ }^{*} P<0.05$. 
A
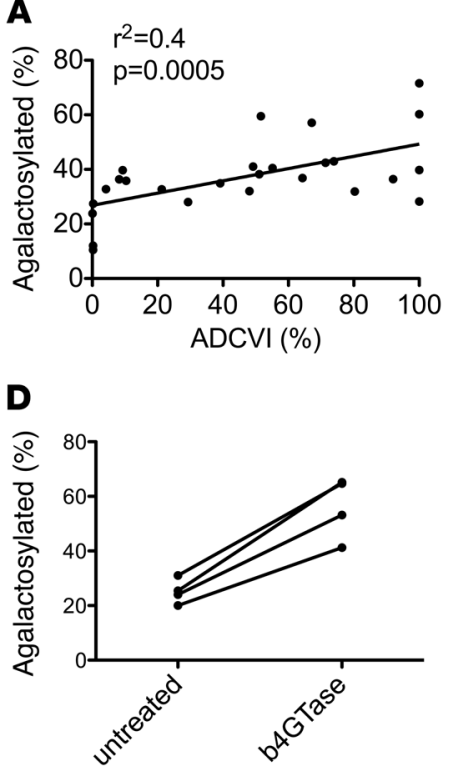
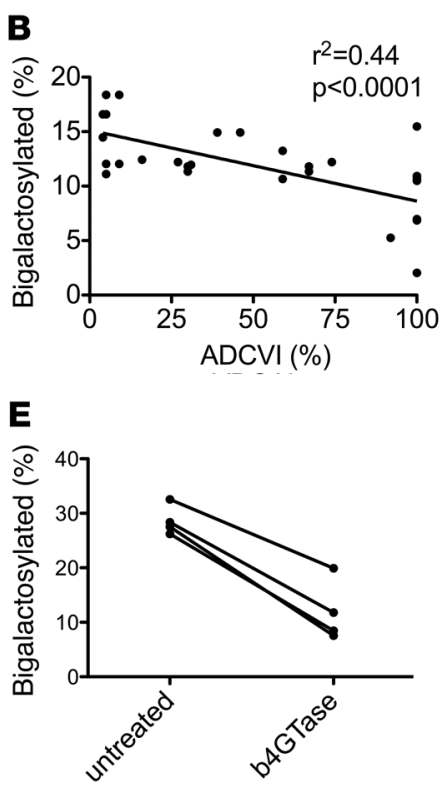
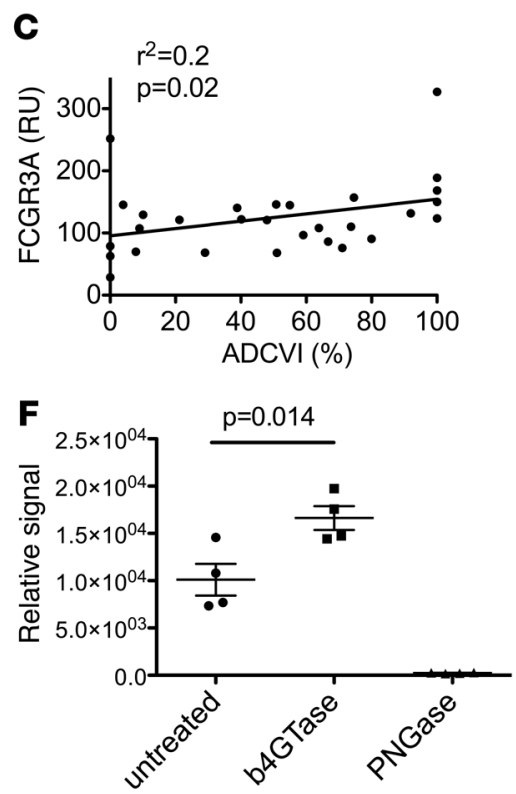

Figure 5

Antiviral activity is associated with specific glycoforms and FCGR3A binding. (A) Total agalactosylated (G0 class) glycans prevalence correlates positively with ADCVI activity. (B) Bigalactosylated (G2 class) glycans prevalence correlates negatively with NK cell ADCVI activity. (C) Bulk plasma antibody affinity for FCGR3A, measured as Biacore response units, correlates positively with ADCVI activity. (D) Impact of enzymatic treatment with a galactoside on agalactosylated glycan and $(\mathbf{E})$ bigalactosylated glycan content. $(\mathbf{F})$ Binding of treated and untreated antibody samples to FCGR3A beads. (A-C and F) Individual symbols represent each subject tested, and horizontal lines represent the median for a given group of clinically related subjects.

the constitutive components of the glycan, including MGAT1, $M G A T 2$, and $A L G 2$ (Figure 6A), which may reflect the hypergammaglobulinemia present in this population. Expression of the fucosyltransferase responsible for core fucosylation, FUT8, was decreased in controllers and treated progressors relative to that in untreated progressors (Figure 6B), while the fucosidase, FUCA2, was enriched in controllers and untreated subjects (Figure 6B). While the biological relevance of FUCA2 expression in B cells is unproven, these expression patterns are nonetheless consistent with the observation of decreased fucosylation in HIV-infected subjects. Expression changes in galactose transferases were also observed (Figure 6C), with HIV-infected subjects exhibiting significantly lower expression of one or both galactose transferases studied. Overall, the alterations in GT expression were consistent with the hypergammaglobulinemia (Figure 6A), decreased fucosylation (Figure 6B), and decreased galactosylation (Figure 6C) observed in the antibodies of infected subjects. Further study of GT expression in HIV-specific B cells is likely to yield additional insight into B cell modulation of antibody activity.

\section{Discussion}

The complexity of antibody functions mediated through FcRs belies the ability of the humoral immune system to tightly regulate recruitment of innate effector cells to rapidly clear the antigens to which they are bound. While only a subset of individuals with progressive HIV infection are able to mount neutralizing antibody activity, individuals that spontaneously control HIV infection generally produce antibodies with a robust capacity to recruit the antiviral activity of the innate immune system, suggesting that innate immune recruiting antibodies, rather than neutralizing antibody responses, may be vital in antiviral control. Therefore, efforts to better understand the characteristics of naturally induced innate immune recruiting antibodies may provide novel information to help improve the functional quality of vaccine-induced antibodies. Significantly, antibodies from HIV-infected subjects vary dramatically in their ability to recruit the cytotoxic functions of NK cells, and these differences could not be attributed to either titer or IgG subclass. Intriguingly, suppression of viral replication, whether spontaneous or mediated by antiretroviral therapy, was associated with more potent effector activity, indicating that specific and global inflammatory cues likely have a complex role in determining antibody glycosylation.

While alterations in antibody glycosylation are present in numerous disease states, we were surprised to find here that (a) despite its long plasma half-life, global plasma IgG glycosylation was significantly perturbed during acute infection; and (b) antibodies from elite controllers, whose plasma generally does not exhibit traditional markers of inflammation, exhibited the most profound IgG glycan skewing. The impact of these global glycosylation changes on humoral immunity is unknown. Furthermore, we found that glycosylation patterns were different between bulk plasma antibody and HIV-specific fractions, indicating that the immune system has evolved means to both globally and specifically, in an antigen-dependent manner, shift antibody function. Defining the signals responsible for the modulation of glycosylation in both global and disease-specific antibody repertoires is likely to be of great clinical relevance in disease states in which antibodies play either protective or pathological roles.

Given the dramatic activity differences in cell-based assays, glycan modulation among subject groups was surprisingly small, leading to the possible conclusion that subtle glycan modulation 
A

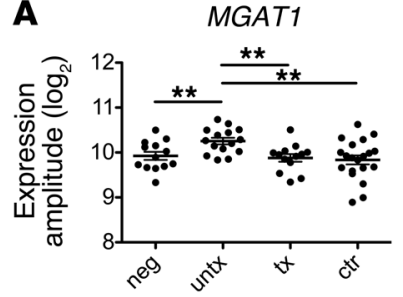

B

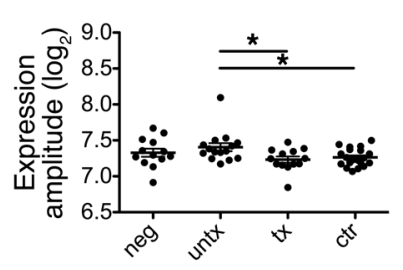

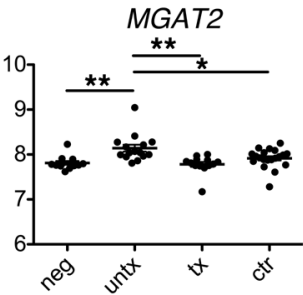

FUCA2

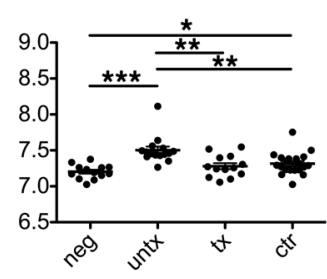

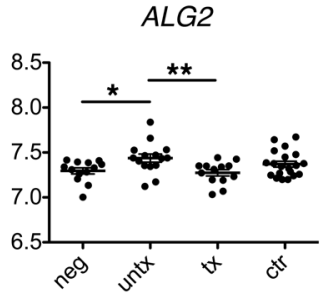

C

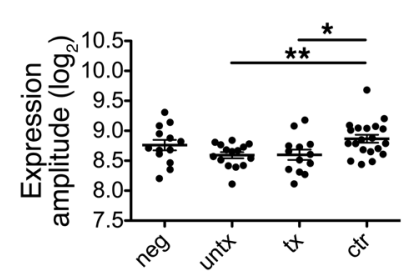

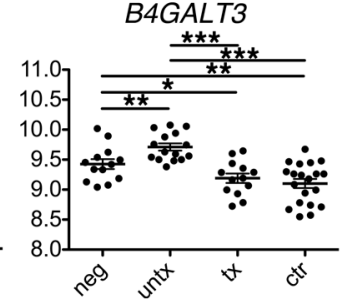

Figure 6

Transcriptional profiling of GTs and glycosidases relevant to IgG glycans in peripheral B cells. (A) GTs involved in synthesis of all antibody glycans, the MGAT1 and MGAT2 enzymes involved in the conversion of oligomannose to complex N-glycans and ALG2 involved in initiating N-linked glycosylation, were upregulated in untreated subjects. (B) Fucosyltransferase FUT8 expression was increased in untreated progressors, while fucosidase FUCA2 was increased in controllers and untreated progressors. (C) Galactose transferases B4GALT1 and B4GALT3 were decreased in HIV-infected subjects. The $y$ axis represents transcript expression relative to a housekeeping gene. Individual symbols represent each subject tested, and horizontal lines represent the median for a given group of clinically related subjects. ${ }^{*} P<0.05 ;{ }^{* \star} P<0.005 ;{ }^{* * *} P<0.0005$.

may lead to significant activity differences, with minor glycan species capable of playing a significant role in overall antibody effector function. This possibility is consistent with other studies in which minor glycan species have been found to be responsible for overall antibody activity (29). Alternatively, because the experimental methods used here could not simultaneously define either the subclasses associated with each glycan structure or assign both Fc glycans present on a given antibody molecule, it is possible that if this level of resolution were possible, perhaps more dramatic differences among subject groups would have been apparent. Furthermore, while some generally applicable principles regarding the effector function of glycans have been determined in studies of monoclonal antibodies, such as the role of fucose in modulating ADCC activity, the context in which these saccharides are present may be important - with fucose having a greater or lesser effect in the context of G0, G1, G2, or bisected glycan chains.

Despite these limitations, a clear association between galactose levels and ADCVI activity was observed. This association was surprising in that increased galactosylation has tended to drive better ADCC in recombinantly produced monoclonal antibodies (26). While there are a number of plausible explanations for this discrepancy, the simplest is that, in naturally produced antibodies, galactosylation covaries with differential sialylation, as sialic acids are incorporated via linkages to terminal galactose groups. Recombinant monoclonal antibodies, typically secreted from animals, and non-B cell types may not exhibit the same covariation in galactose and sialylation or may differ in isomerization patterns due to inherent differences in GT expression or antibody trafficking and processing in the endoplasmic reticulum. Similarly, covariation of oligomannose intermediates may be a confounding factor in studies of recombinant antibodies, whereas these high mannose forms known to have improved ADCC activity are extremely rare in naturally expressed human antibodies. Alternatively, antibody galactosylation has recently been associated with antiinflamma- tory activity following FcgR ligation via interactions with additional binding partners (30). However, here we show that beyond associated minor glycan species changes, agalactosylated antibodies themselves may have an enhanced capacity to bind to FCGR3A, potentially resulting in more potent ADCC/ADCVI activity.

Alterations in antibody glycosylation ultimately result from altered glycan biosynthesis on a cellular level, likely due to changes in the expression of enzymes involved in N-linked glycan biosynthesis or due to the rate of antibody transit through the endoplasmic reticulum prior to secretion. HIV infection was associated with significant shifts in the expression of several GTs and glycosidases, which are involved in multiple steps of glycan synthesis, consistent with the production of a larger population of agalactosylated antibodies. Moreover, elite controllers exhibit an enzyme expression profile marked by changes in enzyme expression, likely resulting in the production of a larger fraction of nonfucosylated antibodies, suggesting that distinct inflammatory cues or differences in the mechanism of B cell priming or education may result in the skewed expression of the enzymes involved in tuning antibody Fc effector functions. Ultimately, understanding the mechanism by which these enzymes are regulated, particularly in HIV-specific B cells, may provide opportunities to begin to define novel strategies by which vaccines could elicit more antiviral innate immune recruiting antibodies.

Overall, we believe these studies highlight the first example of skewed glycosylation of disease-specific antibodies in HIV infection and, more importantly, that these shifts are regulated by both general inflammatory cues as well as antigen-specific fine adjustments, resulting in the production of antibodies with enhanced effector functions. However, whether antibody glycosylation can be regulated permanently in memory B cells or long-lived plasma cells provides an exciting prospect for future vaccine efforts aimed at potentiating the functional capacity of vaccine-induced antibody responses. 


\section{Methods}

Subjects. A total of 90 subjects, including 10 acute/early HIV-infected individuals, 24 chronically infected individuals off therapy, 24 chronically infected subjects receiving treatment, 22 controllers, and 10 HIV-negative individuals, were recruited for antibody analysis studies. The 10 acute/ early HIV-infected subjects, defined as p24-antibody negative or Western blot indeterminate, were recruited within the first 5 months of infection and were followed longitudinally in the absence of antiretroviral therapy. GT expression analysis was conducted on a second group of 61 subjects, and subject characteristics for each study group are reported in Supplemental Table 1. The local and Massachusetts General Hospital Institutional Review Boards approved the study, and each subject provided written informed consent for participation in the study.

Antibody purification. Bulk IgG was purified from $200 \mu \mathrm{l}$ of plasma using a Melon Gel IgG Purification Kit (Thermo Scientific). Briefly, the resin was washed twice with Melon Gel buffer and incubated with diluted plasma on a rotator for 5 minutes to allow for the binding of plasma proteins. Antibodies were spun through the resin, and the concentration was calculated using a Nanodrop spectrophotometer (Thermo Scientific). Following IgG purification, HIV-specific antibodies were affinity purified using a custom amino-link (Pierce) gp120 resin. Each microcolumn was conjugated with $100 \mu \mathrm{g}$ gp120 (Immune Technologies) according to the manufacturer's protocol. Conjugation efficiency was determined to be at least $50 \%$ for all columns prepared. Each column was used to process IgG from a single subject. Briefly, purified subject IgG (2-10 mg) was incubated with the resin prior to washing and low $\mathrm{pH}$ elution. Eluted antibodies were immediately neutralized. Analysis was performed to verify that brief acid exposure did not alter glycan composition and that gp120 was not released from the resin. Antibodies titers were determined by ELISA as described previously (21), and enrichment of gp120-specific antibodies was confirmed by ELISA.

$A D C V I$ assay. The capacity of plasma antibodies to recruit the antiviral activity of NK cells and suppress viral replication was measured using an ADCVI assay (20). Briefly, PBMCs from an HIV-1-negative donor were cultured with a CD3/8 bispecific antibody $(0.3 \mu \mathrm{g} / \mathrm{ml})$ for 4 days in the presence of $50 \mathrm{U} / \mathrm{ml} \mathrm{IL-2}(\mathrm{NIH})$ to produce activated $\mathrm{CD} 4^{+} \mathrm{T}$ cells. On the fourth day, the $\mathrm{CD}^{+} \mathrm{T}$ cells were infected at an MOI of 0.01 with an $\mathrm{R} 5$ viral strain (JRCSF). In parallel, NK cells were purified from the same donor using negative selection (RosetteSep; Stem Cell Technologies). The purified IgG was added to the $\mathrm{CD}^{+}{ }^{+} \mathrm{T}$ cells $(10 \mu \mathrm{g} / \mathrm{ml})$ in duplicate, and NK cells were added to one of the duplicate wells. The supernatant was collected on day 7 , and the level of inhibition was quantified as the difference in $\mathrm{p} 24$ produced in wells containing antibodies/effectors compared with wells containing $\mathrm{CD} 4^{+} \mathrm{T}$ and NK cells alone. Each data point represents the average of 3 independent experiments using different autologous effector/target donors. Supplemental Figure 4 presents the reproducibility of this ADCVI assay across multiple donors assessed on different days. To reduce inhibition due to NK cells alone, only KIR3DS1donor cells were used (31). A subset of antibody samples were treated with PNGaseF (New England Biolabs) to remove N-linked glycans, as previously described (32). The presence of inactivated PNGaseF did not affect the ADCVI assay results.

Antibody-induced NK cell degranulation assay. Intracellular cytokine staining was used to determine whether the IgG purified from the plasma of acutely infected subjects exhibited a differential capacity to promote NK cell cytokine secretion and/or degranulation as a surrogate for NK cell cytolysis (19). Briefly, activated CD4 ${ }^{+} \mathrm{T}$ cells were prepared as described for the ADCVI assay. In parallel, NK cells were purified by negative selection and cultured in media supplemented with $50 \mathrm{U} / \mathrm{ml} \mathrm{IL-2} \mathrm{for} 4$ days. On day 4, the $\mathrm{CD}^{+} \mathrm{T}$ cells were infected with a VSV-pseudotyped R5 virus (JRCSF) for 2 hours and then cultured for an additional 2 days. On day $6, \mathrm{CD}^{+} \mathrm{T}$ cells were then counted and split into duplicate FACS tubes. The purified $\operatorname{IgG}$ was added at $50 \mu \mathrm{g} / \mathrm{ml}$ to each duplicate tube, and NK cells were added to one of the tubes at an effector to target ratio of 1:1. Anti-CD107a-PECy5 (20 $\mu \mathrm{l} / \mathrm{ml}$; BD Biosciences), Brefeldin A $(0.5 \mu \mathrm{g} / \mathrm{ml}$; Sigma-Aldrich), and Golgi-stop $(0.3 \mu \mathrm{g} / \mathrm{ml}$; BD Biosciences $)$ were added, and the tubes were incubated for 6 hours at $37^{\circ} \mathrm{C}$. NK cells, either alone or in the presence of uninfected $\mathrm{CD} 4^{+} \mathrm{T}$ cells, were used as a negative control, and NK cells stimulated with PMA/ionomycin $(1 \mu \mathrm{g} / \mathrm{ml})$ were used as a positive control. Following incubation, the samples were stained with anti-CD3-Pacific Blue, anti-CD56-PE-Cy7, and anti-CD16APC-Cy7 (BD Biosciences) to define NK cells; fixed; permeabilized (FIX$\mathrm{A} / \mathrm{B}, \mathrm{Caltag} /$ Invitrogen); and then stained with anti-IFN- $\gamma$-FITC and anti-TNF- $\alpha$-Alexa 700 (BD Biosciences). The cells were then resuspended in $100 \mu \mathrm{l} 2 \%$ paraformaldehyde-PBS, and data were acquired on a BD LSRII. The proportion of degranulated or cytokine-secreting NK cells was then calculated as follows: (percentage of cells that were positive for the marker of interest / percentage of cells that responded to PMA/ ionomycin) $\times 100$. The background level of NK cell activation by HIV-infected autologous cells was subtracted from the experimental values, and a response was considered positive if the proportion of CD107a-expressing or cytokine-positive cells following stimulation was at least 2-fold greater than the mean of the no plasma controls. Supplemental Figure 5 presents the correlation of ADCVI assay results presented in this study with NK cell degranulation and cytokine secretion.

FoyRIIIa/antibody affinity assays. Surface plasmon resonance was used to measure the strength of the antibody-FCGR3A interaction. Wells on a Biacore CM5 research-grade chip were coated with $5 \mu \mathrm{g}$ recombinant Fc $\gamma$ RIIIa or a control protein (Staphylococcal enterotoxin B, Sigma-Aldrich). Purified bulk IgG was diluted to a concentration of $0.2 \mu \mathrm{g} / \mathrm{ml}$, and the binding affinity was calculated as the peak relative units of binding for each antibody sample normalized to control protein binding. Binding of the monoclonal IgG1 b12 antibody was used as a positive control, and the mutant LALA b12 variant (provided by Dennis Burton, The Scripps Research Institute, La Jolla, California, USA), with impaired FcR binding activity, was used as a negative control.

Generation of $G 0$ antibodies. To generate hypogalactosylated antibody, IgG $(10 \mathrm{ml}$ of $0.250 \mathrm{mg} / \mathrm{ml})$ was isolated from healthy donor plasma using protein $\mathrm{G}$ beads (GE Healthcare) and incubated overnight at $37^{\circ} \mathrm{C}$ in neuraminidase ( $500 \mathrm{U} / \mathrm{ml}$, New England Biologicals), as per manufacturer instructions. The solution containing desialyated IgG was supplemented with $\mathrm{NaCl}$ to a final concentration of $100 \mathrm{mM}$ and incubated overnight at $37^{\circ} \mathrm{C}$ with $\beta$-1,4-galactosidase ( $20 \mathrm{U} / \mathrm{ml}$, New England Biologicals) 3 times. Galactose content was determined by HPLC (33).

FCGR3A binding activity. A customized Luminex assay was developed in which recombinant FCGR3A was covalently conjugated to magnetic beads and incubated in antibody over a dilution range, and bound antibody was quantified with phycoerythrin-conjugated mouse anti-human IgG, in an adaptation of a previously described protocol (34). Binding of a series of Fc domain amino acid point mutants was used to validate that signal in this assay correlated with binding affinity (data not shown).

Glycan analysis by mass spectrometry. Enzymatic release of $\mathrm{N}$-linked glycans was performed according to the method of Küster (35). Oligosaccharides were released from bands containing approximately $10 \mu \mathrm{g}$ target glycoprotein that was excised from Coomassie blue-stained reducing SDS-PAGE gels, washed with alternating water and acetonitrile, and dried in a vacuum centrifuge, followed by rehydration with 100 units/ml of PNGase F (New England Biolabs) and incubation for 12 hours at $37^{\circ} \mathrm{C}$. The enzymatically released $\mathrm{N}$-linked glycans were eluted with water. Aqueous solutions of the glycans were cleaned with a Nafion 117 membrane. 
Positive-ion MALDI-TOF mass spectra were recorded with a Shimadzu AXIMA TOF2 MALDI-TOF/TOF instrument (Shimadzu-Kratos) fitted with delayed extraction and a nitrogen laser $(337 \mathrm{~nm})$. The acceleration voltage was $20 \mathrm{kV}$; the pulsed extraction was optimized for $m / z 1,700$. Samples were prepared by adding $0.5 \mu \mathrm{l}$ of an aqueous solution of the sample to the matrix solution ( $0.3 \mu \mathrm{l}$ of a saturated solution of 2,5-dihydroxybenzoic acid in acetonitrile) on the stainless steel target plate and allowing it to dry at room temperature. The sample/matrix mixture was then recrystallized from ethanol.

Negative-ion ESI mass spectrometry was performed with a Waters Synapt G2 quadrupole-ion mobility-TOF (Q-IMS-TOF) mass spectrometer (Waters MS Technologies) for samples with sufficient material remaining. Sample were dissolved in 1:1 (v/v) water/methanol $(8 \mu \mathrm{l}), 1 \mu \mathrm{l}$ of a $1 \mathrm{M}$ solution of ammonium phosphate was added, and the samples were spun at 3,000 $\mathrm{g}$ in a bench centrifuge for 1 minute. Sample introduction into the mass spectrometer was via Waters thin-wall nanospray capillaries. The ion source temperature was maintained at $120^{\circ} \mathrm{C}$, and fragmentation was performed, with argon, in the instrument's transfer cell. The collision energy was adjusted according to the mass of the parent ions to give an even distribution of fragments across the mass range. Instrument control and data processing were performed with Waters MassLynx software, version 4.1. Fragmentation spectra were interpreted as described by Harvey et al. (36-39).

GT expression profiling. $\mathrm{CD} 3{ }^{-} \mathrm{CD} 19^{+} \mathrm{CD} 27^{+}$cells were separated by magnetic separation (Miltenyi Biotec), and the transcriptional profile of these peripheral B cells was determined using Illumina HumanHT-12 whole-genome expression BeadChips. Briefly, following incubation with magnetic microbeads, cells were thoroughly washed, and a minimum of $5 \times 10^{5}$ pure B cells from each subject was resuspended in RNA later. RNA was extracted using the Qiagen RNeasy Kit (Qiagen). Then, $200 \mathrm{ng}$ of total RNA was amplified using the Ambion Illumina TotalPrep RNA Amplification Kit, and 1,500 ng of the amplified cDNA was hybridized overnight to the BeadChip. After a series of washes, BeadChips were read using the Illumina BeadStation. The resulting images were analyzed using the Illumina BeadStudio software, and internal controls were evaluated for each sample to quality control the data. Data from the samples that passed chip processing quality control was exported and normalized, and expression for a set of 16 GTs with possible activities relevant to the antibody $\mathrm{N}$-linked glycan, as described by the Korean Encyclopedia of Genes and Genomes (KEGG) database $(40,41)$, was extracted. Data presented represent expression amplitude $\left(\log _{2}\right)$.

Subject classification analysis. Three commonly used methods were selected to analyze the information content of the GT expression data set in terms of its ability to predict patient class, including a naive Bayes (NB) classifier, linear discriminant analysis (LDA), and a linear-kernel support vector machine (SVM) approach with a voting mechanism combining binary classifiers for multiclass classification. The classifiers were implemented in the R programming language (42), using the e 1071 package. Classification analysis was conducted using a subset of the GTs identified by the Boruta algorithm (43) as features relevant to discriminating among these subject classes. Features identified as relevant to resolving infected versus uninfected subjects were the expressions of B4GALT4, MGAT2, MGAT4A, and FUCA2. Features with value in resolving all subject classes included ALG2, B4GALT1, B4GALT3, MGAT2, MGAT3, MGAT4A, and FUCA2. The predictive performance of each classifier was assessed with 100 replicates of 5-fold cross validation. Each of the 3 methods (NB, LDA, and SVM) gave similar performance results.

Statistics. An ANOVA with a post-hoc Tukey's test was used to test for differences in the mean among several populations. Spearman correlations were used to examine the interrelatedness of different parameters. $P$ values less than 0.05 were considered significant.

\section{Acknowledgments}

This work was supported by the NIH (R01 AI080289) and the Bill and Melinda Gates Foundation (43307): "HIV Controllers: Implications for Vaccine Design.” M.E. Ackerman was supported by an NIH Harvard University Center for AIDS Research Postdoctoral Fellowship (P30 AI060354-07). I. Choi was supported by NSF grant IIS-0905206. We thank both the International AIDS Vaccine Initiative (IAVI) and the Oxford Glycobiology Institute endowment for financial support. We are particularly grateful to IAVI for an equipment grant to purchase a MALDI mass spectrometer and to Professor J. Scrivens, University of Warwick, for access to the Synapt G2 mass spectrometer. A. Ercan was supported by the Arthritis National Research Foundation and by NIH K12 HD51959. P.A. Nigrovic was supported by the Cogan Family Fund and by NIAID R21-99435.

Received for publication July 9, 2012, and accepted in revised form February 7, 2013.

Address correspondence to: Galit Alter, 400 Technology Square, Cambridge, Massachusetts 02142, USA. Phone: 857.268.7003; Fax: 857.268.7142; E-mail: galter@partners.org.
1. Rerks-Ngarm S, et al. Vaccination with ALVAC and AIDSVAX to prevent HIV-1 infection in Thailand. N Engl J Med. 2009;361(23):2209-2220.

2. Haynes BF, et al. Immune-correlates analysis of an HIV-1 vaccine efficacy trial. $N$ Engl J Med. 2012;366(14):1275-1286.

3. Baum LL, et al. HIV-1 gp120-specific antibody-dependent cell-mediated cytotoxicity correlates with rate of disease progression. J Immunol. 1996;157(5):2168-2173.

4. Forthal DN, Landucci G, Keenan B. Relationship between antibody-dependent cellular cytotoxicity, plasma HIV type 1 RNA, and CD4+ lymphocyte count. AIDS Res Hum Retroviruses. 2001; 17(6):553-561

5. Asmal M, et al. Antibody-dependent cell-mediated viral inhibition emerges after simian immunodeficiency virus SIVmac251 infection of rhesus monkeys coincident with gp140-binding antibodies and is effective against neutralization-resistant viruses. J Virol. 2011;85(11):5465-5475.

6. Hidajat R, et al. Correlation of vaccine-elicited systemic and mucosal nonneutralizing antibody activities with reduced acute viremia following intrarectal simian immunodeficiency virus SIVmac251 challenge of rhesus macaques. J Virol. 2009;83(2):791-801.

7. Florese RH, et al. Contribution of nonneutralizing vaccine-elicited antibody activities to improved protective efficacy in rhesus macaques immunized with Tat/Env compared with multigenic vaccines. Immunol. 2009;182(6):3718-3727.

8. Nimmerjahn F, Ravetch JV. Fc-receptors as regulators of immunity. Adv Immunol. 2007;96:179-204.

9. Strohmeier GR, Brunkhorst BA, Seetoo KF, Bernardo J, Weil GJ, Simons ER. Neutrophil functional responses depend on immune complex valency. J Leukoc Biol. 1995;58(4):403-414.

10. Powell MS, Hogarth PM. Fc receptors. Adv Exp Med Biol. 2008;640:22-34.

11. Shaw GM, Aminoff D, Balcerzak SP, LoBuglio AF. Clustered IgG on human red blood cell membranes may promote human lymphocyte antibody-dependent cell-mediated cytotoxicity. J Immunol. 1980;125(2):501-507.

12. Schroeder HW Jr, Cavacini L. Structure and func- tion of immunoglobulins. J Allergy Clin Immunol. 2010;125(2 suppl 2):S41-S52.

13. Lux A, Nimmerjahn F. Impact of differential glycosylation on IgG activity. Adv Exp Med Biol. 2011;780:113-124.

14. Shields RL, et al. Lack of fucose on human IgG1 $\mathrm{N}$-linked oligosaccharide improves binding to human Fcgamma RIII and antibody-dependent cellular toxicity. J Biol Chem. 2002; 277(30):26733-26740.

15. Anthony RM, Ravetch JV. A novel role for the IgG Fc glycan: the anti-inflammatory activity of sialylated IgG Fcs. J Clin Immunol. 2010;30(suppl 1):S9-S14.

16. Lund J, Takahashi N, Pound JD, Goodall M, Jefferis R. Multiple interactions of IgG with its core oligosaccharide can modulate recognition by complement and human $\mathrm{Fc}$ gamma receptor I and influence the synthesis of its oligosaccharide chains. J Immunol. 1996;157(11):4963-4969.

17. Moore JS, et al. Increased levels of galactose-deficient IgG in sera of HIV-1-infected individuals. AIDS. 2005;19(4):381-389.

18. Troelsen LN, et al. IgG glycosylation changes and 
MBL2 polymorphisms: associations with markers of systemic inflammation and joint destruction in rheumatoid arthritis. J Rheumatol. 2012;39(3):463-469.

19. Alter G, Malenfant JM, Altfeld M. CD107a as a functional marker for the identification of natural killer cell activity. J Immunol Methods. 2004; 294(1-2):15-22.

20. Forthal DN, et al. Antibody-dependent cellular cytotoxicity independently predicts survival in severely immunocompromised human immunodeficiency virus-infected patients. J Infect Dis. 1999;180(4):1338-1341.

21. Banerjee $\mathrm{K}$, et al. IgG subclass profiles in infected HIV type 1 controllers and chronic progressors and in uninfected recipients of Env vaccines. AIDS Res Hum Retroviruses. 2010;26(4):445-458.

22. Harvey DJ, Merry AH, Royle L, Campbell MP, Dwek RA, Rudd PM. Proposal for a standard system for drawing structural diagrams of $\mathrm{N}$ - and O-linked carbohydrates and related compounds. Proteomics. 2009;9(15):3796-3801.

23. Hunt PW, et al. Relationship between T cell activation and CD4+ T cell count in HIV-seropositive individuals with undetectable plasma HIV RNA levels in the absence of therapy. J Infect Dis. 2008;197(1):126-133

24. Brenchley JM, et al. Microbial translocation is a cause of systemic immune activation in chronic HIV infection. Nat Med. 2006;12(12):1365-1371.

25. Sciaranghella G, Tong N, Mahan AE, Suscovich TJ, Alter G. Decoupling activation and exhaustion of $\mathrm{B}$ cells in spontaneous controllers of HIV infection. AIDS. 2013;27(2):175-180.

26. Shinkawa T, et al. The absence of fucose but not the presence of galactose or bisecting $\mathrm{N}$-acetylglu- cosamine of human IgG1 complex-type oligosaccharides shows the critical role of enhancing antibody-dependent cellular cytotoxicity. J Biol Chem. 2003;278(5):3466-3473

27. Umana P, Jean-Mairet J, Moudry R, Amstutz H, Bailey JE. Engineered glycoforms of an antineuroblastoma IgG1 with optimized antibody-dependent cellular cytotoxic activity. Nat Biotechnol. 1999;17(2):176-180.

28. Bolton GR, Ackerman ME, Boesch AW. Separation of nonfucosylated antibodies with immobilized Fc $\gamma$ RIII receptors [published online ahead of print March 6, 2013]. Biotechnol Prog. doi:10.1021/ btpr.1717.

29. Kaneko Y, Nimmerjahn F, Ravetch JV. Anti-inflammatory activity of immunoglobulin $\mathrm{G}$ resulting from Fc sialylation. Science. 2006;313(5787):670-673.

30. Karsten CM, et al. Anti-inflammatory activity of IgG1 mediated by Fc galactosylation and association of FcgammaRIIB and dectin-1. Nat Med. 2012;18(9):1401-1406.

31. Alter G, et al. Differential natural killer cell-mediated inhibition of HIV-1 replication based on distinct KIR/ HLA subtypes. J Exp Med. 2007;204(12):3027-3036.

32. Ackerman ME, et al. A robust, high-throughput assay to determine the phagocytic activity of clinical antibody samples. J Immunol Methods. 2011;366(1-2):8-19.

33. Ercan A, et al. Multiple juvenile idiopathic arthritis subtypes demonstrate proinflammatory IgG glycosylation. Arthritis Rheum. 2012;64(9):3025-3033.

34. Brown EP, et al. High-throughput, multiplexed IgG subclassing of antigen-specific antibodies from clinical samples. J Immunol Methods. 2012; 386(1-2):117-123.
35. Küster B, Wheeler SF, Hunter AP, Dwek RA, Harvey $\mathrm{DJ}$. Sequencing of $\mathrm{N}$-linked oligosaccharides directly from protein gels: in-gel deglycosylation followed by matrix-assisted laser desorption/ ionization mass spectrometry and normal-phase high-performance liquid chromatography. Anal Biochem. 1997;250(1):82-101.

36. Harvey DJ. Fragmentation of negative ions from carbohydrates: part 3. Fragmentation of hybrid and complex N-linked glycans. J Am Soc Mass Spectrom. 2005;16(5):647-659.

37. Harvey DJ. Fragmentation of negative ions from carbohydrates: part 2. Fragmentation of high-mannose N-linked glycans. J Am Soc Mass Spectrom. 2005;16(5):631-646.

38. Harvey DJ. Fragmentation of negative ions from carbohydrates: part 1 . Use of nitrate and other anionic adducts for the production of negative ion electrospray spectra from $\mathrm{N}$-linked carbohydrates. J Am Soc Mass Spectrom. 2005;16(5):622-630.

39. Harvey DJ, Royle L, Radcliffe CM, Rudd PM, Dwek RA. Structural and quantitative analysis of $\mathrm{N}$-linked glycans by matrix-assisted laser desorption ionization and negative ion nanospray mass spectrometry. Anal Biochem. 2008;376(1):44-60.

40. Hashimoto K, et al. KEGG as a glycome informatics resource. Glycobiology. 2006;16(5):63R-70R.

41. Ogata H, Goto S, Sato K, Fujibuchi W, Bono H, Kanehisa M. KEGG: Kyoto Encyclopedia of Genes and Genomes. Nucleic Acids Res. 1999;27(1):29-34.

42. R Development Core Team. R: A Language and Environment for Statistical Computing. Vienna, Austria: R Foundation for Statistical Computing; 2011.

43. Kursa MB, Rudnicki WR. Feature Selection with the Boruta Package. J Stat Softw. 2010;36(11):1-13. 